JOURNAL OF SYNCHROTRON RADIATION

ISSN 1600-5775

Received 3 November 2016 Accepted 28 December 2016

Edited by J. F. van der Veen

Keywords: inelastic x-ray scattering; X-ray Raman spectroscopy; beamline; spectrometer; ESRF.

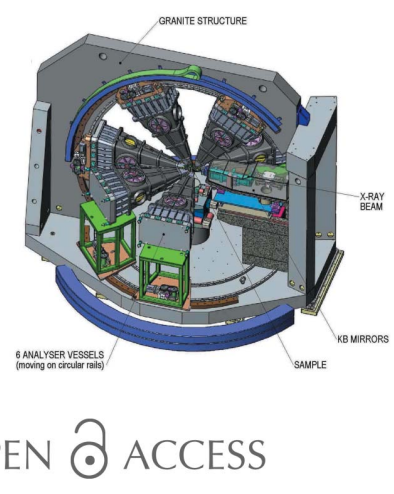

\section{A large-solid-angle X-ray Raman scattering spectrometer at ID20 of the European Synchrotron Radiation Facility}

\author{
S. Huotari, ${ }^{a, b *}$ Ch. J. Sahle, ${ }^{a}$ Ch. Henriquet, ${ }^{a}$ A. Al-Zein, ${ }^{a, c}$ K. Martel, ${ }^{a}$ \\ L. Simonelli, ${ }^{a, d}$ R. Verbeni, ${ }^{a}$ H. Gonzalez, ${ }^{a}$ M.-C. Lagier, ${ }^{a}$ C. Ponchut, ${ }^{a}$ \\ M. Moretti Sala, ${ }^{a}$ M. Krisch ${ }^{a}$ and G. Monaco ${ }^{a, e}$
}

${ }^{\mathbf{a}}$ ESRF - The European Synchrotron, CS40220, 38043 Grenoble Cedex 9, France, ${ }^{\mathbf{b}}$ Department of Physics, PO Box 64, University of Helsinki, 00014 Helsinki, Finland, 'Department of Physics, Faculty of Science, Beirut Arab University, Beirut 11072809, Lebanon, 'dALBA Synchrotron Light Facility, Carrer de la Llum 2-26, 08290 Cerdanyola del Vallés, Barcelona, Spain, and ${ }^{\mathbf{e}}$ Dipartimento di Fisica, Università di Trento,

via Sommarive 14, 38123 Povo (TN), Italy. *Correspondence e-mail: simo.huotari@helsinki.fi

An end-station for X-ray Raman scattering spectroscopy at beamline ID20 of the European Synchrotron Radiation Facility is described. This end-station is dedicated to the study of shallow core electronic excitations using non-resonant inelastic X-ray scattering. The spectrometer has 72 spherically bent analyzer crystals arranged in six modular groups of 12 analyzer crystals each for a combined maximum flexibility and large solid angle of detection. Each of the six analyzer modules houses one pixelated area detector allowing for X-ray Raman scattering based imaging and efficient separation of the desired signal from the sample and spurious scattering from the often used complicated sample environments. This new end-station provides an unprecedented instrument for X-ray Raman scattering, which is a spectroscopic tool of great interest for the study of low-energy X-ray absorption spectra in materials under in situ conditions, such as in operando batteries and fuel cells, in situ catalytic reactions, and extreme pressure and temperature conditions.

\section{Introduction}

$\mathrm{X}$-ray Raman scattering (XRS) spectroscopy is a versatile tool for studying shallow X-ray absorption edges using hard $\mathrm{X}$-rays. It has proven an invaluable technique for the study of electronic excitations in a variety of sample systems such as crystals (Mattila et al., 2005; Sternemann et al., 2008; Pylkkänen et al., 2010; Huotari et al., 2012; Nyrow et al., 2014a,b; Tse et al., 2014; Pascal et al., 2014; Galambosi et al., 2006; Conrad et al., 2009; Moretti Sala et al., 2014), liquids (Wernet et al., 2004; Pylkkänen et al., 2011; Sahle et al., 2013, 2016a; Juurinen et al., 2013, 2014; Niskanen et al., 2015) and gases (Sakko et al., 2011; Inkinen et al., 2013; Zhu et al., 2011). The inherent properties of hard X-rays used for this technique render XRS perfectly suitable for the study of soft X-ray absorption spectroscopy (XAS) or the corresponding counterpart of electron-energy-loss spectroscopy (EELS), often named energy-loss near-edge structure (Egerton, 2011) (ELNES), spectra from complex sample environments that prohibit other probes such as soft X-rays or electrons. XRS provides a truly bulk-sensitive probe for samples inside, for example, in situ catalytic reactors, in operando electrochemical cells, and high-pressure diamond anvil cells. Over the past decades, XRS has been applied to solve geoscientific questions by studying shallow core edges under extreme 
pressure and temperature conditions (Sahle et al., 2013; Mao et al., 2003; Lee et al., 2008; Rueff \& Shukla, 2010; Tse et al., 2011; Shieh et al., 2013; Ding et al., 2014), follow chemical reactions in situ (Sahle et al., 2016a; Miedema et al., 2012; Inkinen et al., 2015) and study liquid samples under well defined thermodynamic conditions (Pylkkänen et al., 2011; Juurinen et al., 2013, 2014; Niskanen et al., 2015; Sahle et al., 2016b). It can even be used as a contrast mechanism for three-dimensional imaging (Huotari et al., 2011; Sahle et al., 2017a,b).

Besides true bulk sensitivity, scattering of a hard X-ray photon by an electron can lead to a significant amount of exchanged momentum. This can be exploited to study the full electronic structure of materials beyond the dipole limit (Mattila et al., 2005; Krisch et al., 1997; Soininen et al., 2005). Furthermore, the non-resonant nature of the XRS process renders the signal independent of the de-excitation channel, which leads to negligible self-absorption effects and a more reliable spectral shape in XRS than in the complementary soft- or tender-XAS techniques. Using XRS, both the XANES and the EXAFS (Huotari et al., 2012; Bergmann et al., 2007; Hiraoka et al., 2013, 2016) regions are accessible.

An obvious drawback of XRS is the orders-of-magnitude weaker scattering cross section in comparison with the probability for photoelectric absorption. This can be compensated for by using light sources with a very high brilliance and efficient signal collection. This has been the design target of high-efficiency XRS end-stations around the world (see, for example, Sokaras et al., 2012; Verbeni et al., 2009; Fister et al., 2006; Cai et al., 2003) and it has also been the guiding motive for the design of the spectrometer presented here.

In this article, we present the new XRS end-station currently installed and commissioned at the inelastic X-ray scattering spectroscopy beamline ID20 of the European Synchrotron Radiation Facility (ESRF, Grenoble, France). First, we briefly introduce the theoretical background necessary for XRS in $\S 2$, then we will give a detailed description of this new large-solid-angle spectrometer and provide its key characteristics in $\S 3$. We will further describe in brief the data analysis (\$4), show some representative data taken with the end-station (§5), and provide conclusions and an outlook in $\S 6$.

\section{Theoretical background}

The measured signal in XRS, and in non-resonant inelastic $\mathrm{X}$-ray scattering (IXS) in general, is proportional to the double differential cross section (Schülke, 2007),

$$
\frac{\mathrm{d}^{2} \sigma}{\mathrm{d} \Omega \mathrm{d} \omega}=\left(\frac{\mathrm{d} \sigma}{\mathrm{d} \Omega}\right)_{\mathrm{Th}} S(\mathbf{q}, \omega)
$$

which consists of the Thomson scattering cross section,

$$
\left(\frac{\mathrm{d} \sigma}{\mathrm{d} \Omega}\right)_{\mathrm{Th}}=r_{\mathrm{e}}^{2}\left(\frac{\omega_{2}}{\omega_{1}}\right)\left(\hat{\mathbf{e}}_{1} \cdot \hat{\mathbf{e}}_{2}\right),
$$

multiplied by the dynamic structure factor

$$
S(\mathbf{q}, \omega)=\sum_{\mathrm{i}, \mathrm{f}} p_{\mathrm{i}}|\langle\mathrm{f}|\exp (i \mathbf{q} \cdot \mathbf{r})| \mathrm{i}\rangle|^{2} \delta\left(E_{\mathrm{i}}-E_{\mathrm{f}}+\omega\right)
$$

Here, $\omega$ is the energy transferred to the sample, $\omega_{1}$ is the energy of the incident photons, $\omega_{2}$ is the energy of the scattered photons, $r_{\mathrm{e}}$ is the classical electron radius, and $\hat{\mathbf{e}}_{1}\left(\hat{\mathbf{e}}_{2}\right)$ is the polarization vector of the incident (scattered) photons. Further, $|i\rangle$ is the electron initial state (typically a shallow or low-energy core level) weighted by its probability $p_{\mathrm{i}}$ and the summation is over all possible final states $|\mathrm{f}\rangle$. The dynamic structure factor is the physical quantity that contains all information obtainable from the sample via non-resonant inelastic X-ray scattering. A few curious facts can be drawn from the above formalism. First of all, in the limit of small momentum transfer $|\mathbf{q}|$, the operator can be expanded as $\exp (i \mathbf{q} \cdot \mathbf{r}) \approx 1+i \mathbf{q} \cdot \mathbf{r}-(\mathbf{q} \cdot \mathbf{r})^{2} / 2+\ldots$ The second term is a dipolar operator and dominates in the limit $\mathbf{q} \cdot \mathbf{r}<1$. It has been shown by Mizuno \& Ohmura (1967) that in this limit the dynamic structure factor is directly related to the photoabsorption spectrum. However, interesting phenomena can occur since the transferred momentum can reach large values in comparison with the radial extent of the wavefunction $|\mathrm{i}\rangle$. Then, the higher-order, i.e. non-dipolar, terms in the Taylor expansion can become dominant. For instance, transitions from an $s$-type symmetry initial state to $s$-type final states become allowed. This has been used in the investigations of dipole-forbidden excitons in the $K$-edges of elements (Hämäläinen et al., 2002) as well as in the studies of preresonances of rare-earth ions (Gordon et al., 2007; Huotari et al., 2015). This $q$-dependence can be exploited to measure natural linear dicroism in cubic systems (Gordon et al., 2009). Other experimental results demonstrate the high potential of XRS as a bulk-sensitive technique for the characterization of the electronic properties of actinide materials by studying the $5 d-5 f$ electric multipole transitions at high momentum transfer (Caciuffo et al., 2010). A highly interesting use of XRS is furthermore the study of low-energy core-electron excitation spectra in complex environments (high-pressure diamond anvil cells, in situ chemical reaction cells, etc.) in which softXAS or EELS studies are not possible due to the highly absorbing sample containers (Sahle et al., 2013; Lee et al., 2008).

\section{Spectrometer}

The large-solid-angle XRS spectrometer has been constructed and commissioned as one of two spectrometers on port ID20 within the framework of the ESRF Upgrade Phase I. The new beamline and the spectrometer are an upgrade to one of the two branches of the inelastic scattering beamline previously located on port ID16 (Verbeni et al., 2009).

ESRF operates a $6 \mathrm{GeV}$ storage ring with a maximum ring current of $200 \mathrm{~mA}$. The straight section for ID20 contains one $26 \mathrm{~mm}$-period and three revolver undulators that are switchable between $32 \mathrm{~mm}$ and $26 \mathrm{~mm}$ periods. The optics that prepare the incident beam are the same as for the second spectrometer on ID20, which is dedicated to high-resolution 
inelastic X-ray scattering and which will be described elsewhere (Moretti Sala et al., 2017). The incident-photon beam is collimated in the vertical plane by a white-beam mirror, which also serves as a heat-load filter for a liquid-nitrogen-cooled double-crystal $\mathrm{Si}(111)$ pre-monochromator. The pre-monochromator can work alone as a fixed-exit monochromator or be coupled with a post-monochromator to form a fixed-exit ensemble. Various post-monochromator options are available, e.g. a $\operatorname{Si}(311)$ channel-cut, or four-bounce $\operatorname{Si}(n n n)(n=3,4,5)$ or $\mathrm{Si}(n n 0)(n=4,6,8)$ post-monochromators. Also, a backscattering $\operatorname{Si}(n n n)$ channel-cut monochromator is available for highly specialized ultra-high-energy-resolution applications. Downstream, a toroidal mirror focuses on a secondary source at a distance of $53 \mathrm{~m}$ from the source and $13.7 \mathrm{~m}$ from the sample position. This secondary source is refocused by a Kirkpatrick-Baez (KB) mirror ensemble to a $8 \mu \mathrm{m} \times 16 \mu \mathrm{m}$ spot size $(\mathrm{V} \times \mathrm{H})$ at the sample position. The working distances of the KB mirror pair are $1.0 \mathrm{~m}$ and $0.5 \mathrm{~m}$. Typical photon fluxes at the sample position without post-monochromator are $4 \times 10^{12}, 7 \times 10^{13}$ and $5 \times 10^{13}$ photons s $^{-1}$ at $6.5 \mathrm{keV}, 9.7 \mathrm{keV}$ and $12.9 \mathrm{keV}$, respectively. With a $\mathrm{Si}(311)$ channel-cut, these numbers are reduced by a factor of seven. For details on the energy resolution, see subsequent subsections.

The spectrometer is optimized for non-resonant IXS experiments with an energy resolution of $\sim 0.3-2.0 \mathrm{eV}$, and covers a large solid angle for the collection of the scattered radiation. The leading goal was to place a large number of analyzer crystals in positions corresponding to well defined momentum transfers $q$. These values of $q$ are tunable to match the requirements of the sample environment and the scientific problem at hand.

Since XRS is a non-resonant IXS process, the exact values of incident and scattered photon energies $\left(\omega_{1}\right.$ and $\left.\omega_{2}\right)$ are less relevant, and the measured quantity depends only on the energy transfer $\omega=\omega_{1}-\omega_{2}$. Therefore, a certain freedom exists for the choice of the incident energy. Depending on the exact setup, most XRS spectrometers use incident photon energies between 5 and $20 \mathrm{keV}$. The design of non-resonant IXS spectrometers can be made simpler than those made for resonant IXS, since $\omega_{2}$ can be kept fixed and only $\omega_{1}$ is scanned.

The entire spectrometer, shown in Fig. 1, is mounted on a massive granite support, which also holds the $\mathrm{KB}$ mirror assembly. The spectrometer is based on Johann-type analyzer crystals. The analyzers are attached to motorized modular units, three of which operate in the horizontal scattering plane, and three units in the vertical scattering plane. Each unit contains 12 spherically bent $\mathrm{Si}(n n 0)$ crystals of $100 \mathrm{~mm}$ diameter (three rows of four analyzers each) with a bending radius of $1.0 \mathrm{~m}$, for a total of 72 crystals. Each analyzer goniometer is equipped with three motorized movements $(\Theta, \chi$ and a translation along the incident beam direction, $t x$ ). The analyzers are cut to $80 \mathrm{~mm}$ active diameter in one direction in order to minimize strain due to angular compression (see following section) and in order to maximize the solid-angle of collection while keeping a compact form for the analyzer
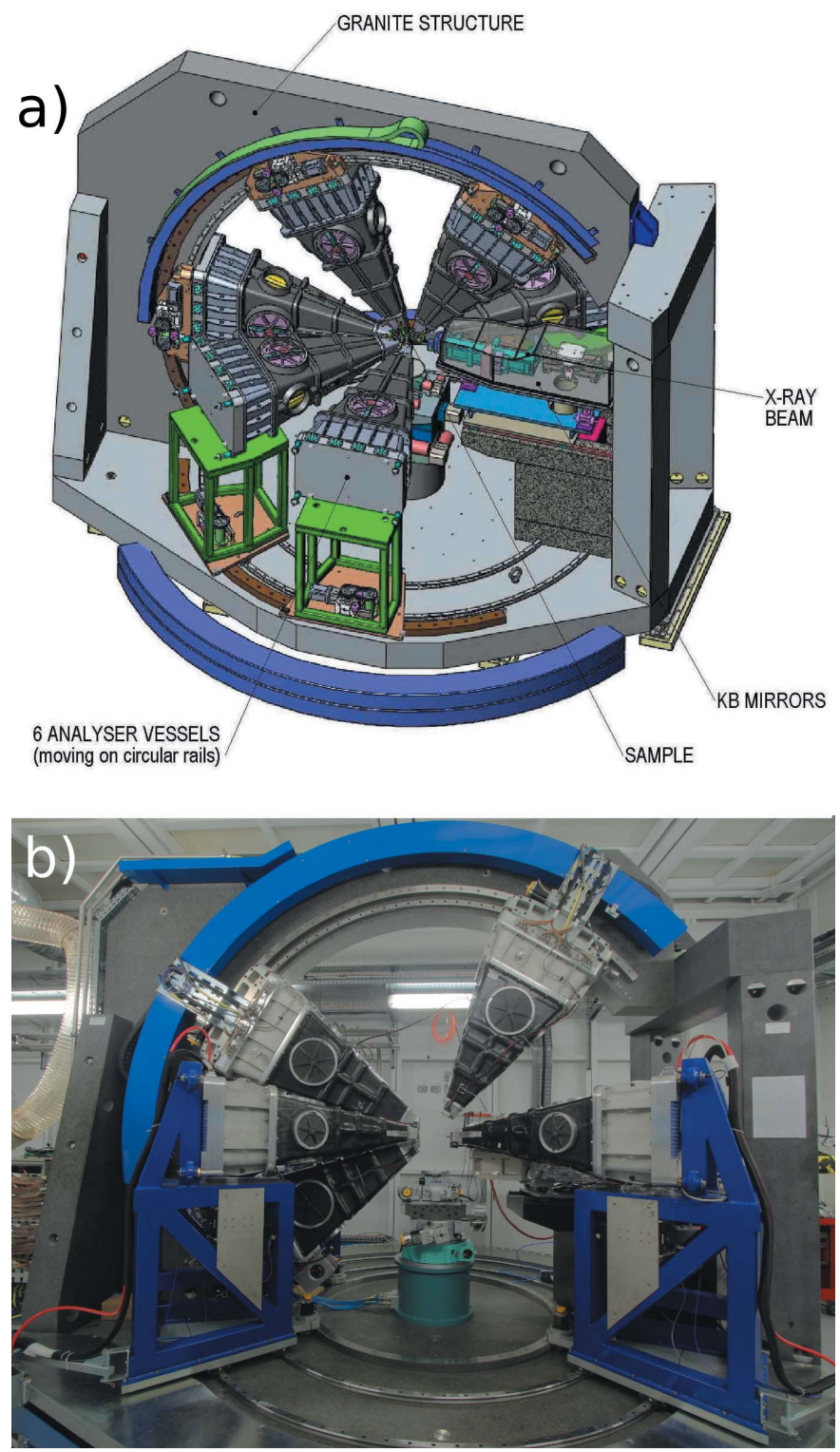

Figure 1

(a) Schematic view of the entire spectrometer assembly consisting of six independently movable crystal analyzer chambers, each hosting 12 spherically bent crystal analyzers. Three chambers are movable in the vertical plane and three in the horizontal plane. All analyzer chambers, the sample stage and the KB mirror system rest on a common granite plate. (b) Photograph of the spectrometer as installed in the experimental hutch.

array. A schematic drawing of a single analyzer unit is shown in Fig. 2(a). Fig. 2(b) shows a single analyzer mount with its two rotational and one translational degrees of freedom. Each module is equipped with a lightweight composite carbon fiber vacuum chamber, which is operated in a rough vacuum of approximately $1 \mathrm{mbar}$ in order to minimize absorption and parasitic scattering from air. At the exit of the vacuum chambers, a single-chip Maxipix (Ponchut et al., 2011) detector head implementing a Timepix readout chip (Llopart et al., 2007) is mounted as close as possible to the sample position in order to allow the spectrometer to operate in a near-backscattering geometry. 
a)

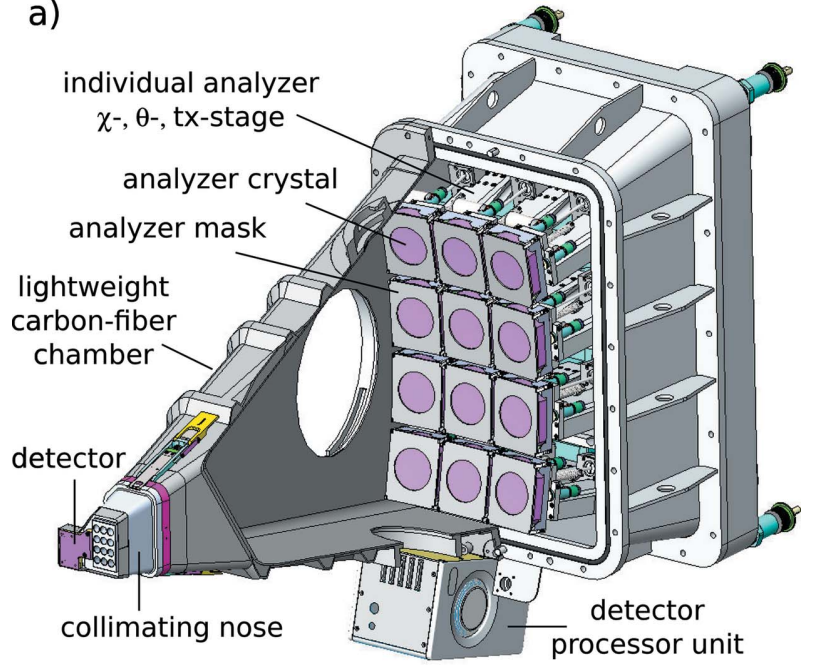

b)

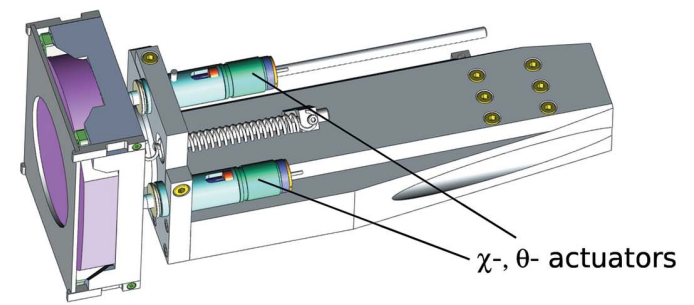

Figure 2

(a) Sketch of an analyzer module hosting 12 analyzer crystals on a $1 \mathrm{~m}$ Rowland circle. (b) Technical drawing of an individual analyzer crystal mount with its two rotational and one translational degrees of freedom.

The naming convention for the six analyzer units are vertical down (VD), vertical up (VU), horizontal left (HL) and horizontal right (HR) modules in the forward direction, and vertical back (VB) and horizontal back (HB) modules for the backscattering ones. However, for the selection of the required momentum transfer range, the scattering angles of all modules can be adjusted individually and a variety of configurations is possible; a typical analyzer unit arrangement in a transmission measurement using, for example, a liquid jet or liquid flow could have four units (two horizontal, two vertical) at scattering angles in the forward direction (for example, centered around $2 \Theta=35^{\circ}$ ) and two units in the reflection geometry at large scattering angles (one horizontal and one vertical unit at $\left.2 \Theta=122^{\circ}\right)$. This allows collecting data at low values of $q$ (for Si660 analyzer reflection, $q_{\min } \simeq 1.5 \AA^{-1}$ ) and high values of $q$ (for Si660 reflection, $q_{\max } \simeq 9.5 \AA^{-1}$ ) simultaneously while maximizing the collected solid angle at both $q$ values.

\subsection{Energy resolution}

The main contributions to the overall energy resolution arise from the incident beam bandwidth, the analyzer crystal bandpass and the so-called off-Rowland contribution. In the following, we will briefly describe the origin for the contributions related to the spectrometer.

Most geometrical factors to the energy resolution scale as $\cot \Theta_{B}$, where $\Theta_{B}$ is the Bragg angle of the analyzer reflection,
Table 1

Calculated strain-induced analyzer crystal contributions to the resolution function in $\mathrm{eV}$ with masks with varying diameter and at different reflection orders. These values do not depend strongly on the exact Bragg angle.

\begin{tabular}{lcllll}
\hline Reflection & $\begin{array}{l}\text { Energy } \\
(\mathrm{keV})\end{array}$ & $40 \mathrm{~mm}$ & $60 \mathrm{~mm}$ & $80 \mathrm{~mm}$ & $\begin{array}{l}\text { No } \\
\text { mask }\end{array}$ \\
\hline $\mathrm{Si}(4,4,0)$ & 6.46 & 0.1 & 0.3 & 0.5 & 0.7 \\
$\mathrm{Si}(6,6,0)$ & 9.69 & 0.3 & 0.5 & 0.7 & 1.0 \\
$\mathrm{Si}(8,8,0)$ & 12.92 & 0.8 & 1.0 & 1.2 & 1.5 \\
$\mathrm{Si}(10,10,0)$ & 16.15 & 1.5 & 1.6 & 1.8 & 2.0 \\
\hline
\end{tabular}

and it is therefore beneficial to design the instrument such that $\Theta_{B}$ is as close to $90^{\circ}$ as possible. However, the fact that all analyzer crystals are to be illuminated by the inelastically scattered radiation from the sample and because of space constraints for extended sample environments, $\Theta_{B}$ is necessarily smaller than $90^{\circ}$. In particular, the Bragg angles for the three rows of analyzers within one analyzer module will differ slightly. For example, for the most commonly used configuration with a free sample-to-detector distance of $140 \mathrm{~mm}$, the Bragg angle values for the three rows of analyzer crystals are $87.8^{\circ}, 88.3^{\circ}$ and $88.9^{\circ}$ (assuming an arrangement of the analyzer foci on the detector active area that results in as similar Bragg angles for all analyzers as possible). For a distance of $75 \mathrm{~mm}$ between the detector active surface and the sample, the Bragg angles are increased to $88.5^{\circ}, 88.7^{\circ}$ and $88.9^{\circ}$, respectively, resulting in a slightly improved overall energy resolution.

3.1.1. Analyzer resolution function. The spectral broadening due to the elastic deformation of a bent analyzer crystal is discussed in detail elsewhere (Verbeni et al., 2005; Honkanen et al., 2014a). The currently used analyzer crystals are based on $300 \mu \mathrm{m}$-thick anodically bonded Si wafers that are not stress relief cut (so-called bent-dicing). Such stress relief cuts have been shown to decrease the contribution of socalled angular compression (Honkanen et al., 2014a,b), which is currently the main contribution to the crystal analyzer energy resolution. Complete elimination of this angular compression would lead to an energy resolution of the analyzer crystals as described by the theory of Takagi and Taupin (Takagi, 1962; Taupin, 1964). Relief cuts to minimize the contribution to the energy resolution by this angular compression can be implemented in the future. Strain-induced contributions to the energy resolution can furthermore be reduced by using apertures in front of the analyzers (i.e. masks) and thus reducing the illuminated crystal area, i.e. reducing the solid angle of detection. At present, available choices for mask apertures are circular masks with diameters of $40 \mathrm{~mm}, 60 \mathrm{~mm}$ and $80 \mathrm{~mm}$. Calculated contributions to the energy resolution induced by the strain in the analyzer crystal are summarized for different analyzer apertures in Table 1.

3.1.2. Off-Rowland contribution. Since the spectrometer should operate at analyzer Bragg angles as close to $90^{\circ}$ as possible and, at the same time, it is necessary to reserve a certain amount of space for the sample environment, the 
Table 2

Calculated off-Rowland contribution to the resolution function in $\mathrm{eV}$ with masks with varying diameter and at different reflection orders. These are values for the middle row of analyzers (there is in addition one row of analyzers with slightly higher values and one row with slightly lower ones).

\begin{tabular}{llllll}
\hline $\begin{array}{l}\text { Sample } \\
\text { distance }\end{array}$ & Reflection & $40 \mathrm{~mm}$ & $60 \mathrm{~mm}$ & $80 \mathrm{~mm}$ & $\begin{array}{l}\text { No } \\
\text { mask }\end{array}$ \\
\hline \multirow{4}{*}{$75 \mathrm{~mm}$} & $\mathrm{Si}(4,4,0)$ & 0.11 & 0.17 & 0.23 & 0.28 \\
& $\mathrm{Si}(6,6,0)$ & 0.18 & 0.27 & 0.37 & 0.46 \\
& $\mathrm{Si}(8,8,0)$ & 0.23 & 0.34 & 0.45 & 0.56 \\
& $\mathrm{Si}(10,10,0)$ & 0.28 & 0.42 & 0.56 & 0.70 \\
\hline Sample & & & & & \\
distance & Reflection & $40 \mathrm{~mm}$ & $60 \mathrm{~mm}$ & $80 \mathrm{~mm}$ & mask \\
\hline \multirow{5}{*}{$140 \mathrm{~mm}$} & $\mathrm{Si}(4,4,0)$ & 0.22 & 0.33 & 0.44 & 0.55 \\
& $\mathrm{Si}(6,6,0)$ & 0.36 & 0.54 & 0.76 & 0.90 \\
& $\mathrm{Si}(8,8,0)$ & 0.44 & 0.66 & 0.88 & 1.10 \\
& $\mathrm{Si}(10,10,0)$ & 0.55 & 0.83 & 1.10 & 1.38 \\
\hline
\end{tabular}

detector is placed inside the Rowland circle by a distance of $2 z$. In order to maintain the foci of the analyzer crystals on the detector, the analyzers are moved away from the sample correspondingly by a distance $z$. This causes the Bragg angles to vary across the analyzer and thus generates a dispersion given approximately by

$$
\frac{\Delta E}{E}=\frac{z D}{R^{2}} \cot \Theta_{\mathrm{B}}
$$

Here, $D$ is the size of the analyzer crystal in the dispersive direction ( $80 \mathrm{~mm}$ in the current case). This dispersion is called the off-Rowland contribution to the resolution function and it may become an important limiting factor in the total resolving power. To minimize the off-Rowland contribution, the relative offset $z / R$ and the individual analyzer opening $D / R$ should be minimized and $\Theta_{\mathrm{B}}$ kept as close to $90^{\circ}$ as possible. However, the simultaneous requirements of a sufficient space for sample environments and a high energy resolution may become mutually excluding and certain compromises are necessary.

At present, there are two choices of sample-to-detector distances to adopt for different sample environments and requirements for energy resolution. The different choices are realised by two different detector housings (see next section) leading to sample-to-detector distances of $2 z=75 \mathrm{~mm}$ and $2 z=140 \mathrm{~mm}$. The off-Rowland contributions of these different choices are summarized in Table 2.

3.1.3. Energy resolution of the incident X-rays. The monochromator configurations available are a cryogenically cooled Si(111) pre-monochromator only or a combination of this pre-monochromator with a variety of post-monochromators that were described earlier in this article. With the utilized spherically bent analyzers crystals, the $\mathrm{Si}(311)$ channel-cut monochromator matches the spectrometer resolution best so it is the most commonly used option for a postmonochromator. Contributions to the overall energy resolution due to the incident bandwidth are summarized for the two most useful monochromator configurations [Si(111) pre-
Table 3

Calculated incident bandwidth contribution to the overall resolution function with different monochromator ensembles and different reflection orders (Shvydko, 2004). Values are in eV.

\begin{tabular}{lll}
\hline Energy & $\begin{array}{l}\mathrm{Si}(111) \\
\text { monochromator }\end{array}$ & $\begin{array}{l}\mathrm{Si}(311) \\
\text { channel-cut }\end{array}$ \\
\hline $\mathrm{Si}(4,4,0)$ & 0.71 & 0.25 \\
$\mathrm{Si}(6,6,0)$ & 1.07 & 0.40 \\
$\mathrm{Si}(8,8,0)$ & 1.42 & 0.54 \\
$\mathrm{Si}(10,10,0)$ & 1.78 & 0.67 \\
\hline
\end{tabular}

Table 4

Measured FWHM of the spectrometer resolution functions at different reflection orders, monochromator settings and sample-detector distances. All results were obtained using $60 \mathrm{~mm}$ masks. The reported values are in $\mathrm{eV}$. The calculated (Calc.) values are the root mean squares of the values reported in Tables 1, 2 and 3.

\begin{tabular}{lrllll}
\hline Reflection & $\begin{array}{l}\text { Energy } \\
(\mathrm{keV})\end{array}$ & $\begin{array}{l}\mathrm{Si}(111)+ \\
\mathrm{Si}(311)\end{array}$ & Calc. & $\mathrm{Si}(111)$ & Calc. \\
\hline $2 z=75 \mathrm{~mm}$ & & & & & \\
$\mathrm{Si}(4,4,0)$ & 6.46 & $0.32 \pm 0.01$ & 0.42 & $0.89 \pm 0.01$ & 0.79 \\
$\mathrm{Si}(6,6,0)$ & 9.69 & $0.65 \pm 0.02$ & 0.69 & $1.39 \pm 0.02$ & 1.21 \\
$\mathrm{Si}(8,8,0)$ & 12.92 & $1.15 \pm 0.02$ & 1.18 & $2.03 \pm 0.01$ & 1.77 \\
$\mathrm{Si}(10,10,0)$ & 16.15 & $1.72 \pm 0.08$ & 1.78 & $2.62 \pm 0.02$ & 2.43 \\
& & & & & \\
$2 z=140 \mathrm{~mm}$ & & & & & \\
$\mathrm{Si}(4,4,0)$ & 6.46 & $0.42 \pm 0.02$ & 0.51 & $0.90 \pm 0.02$ & 0.84 \\
$\mathrm{Si}(6,6,0)$ & 9.69 & $0.73 \pm 0.03$ & 0.84 & $1.49 \pm 0.05$ & 1.30 \\
$\mathrm{Si}(8,8,0)$ & 12.92 & $1.24 \pm 0.03$ & 1.31 & $2.08 \pm 0.01$ & 1.86 \\
$\mathrm{Si}(10,10,0)$ & 16.15 & $1.77 \pm 0.10$ & 1.92 & $2.70 \pm 0.04$ & 2.53 \\
\hline
\end{tabular}

Table 5

Measured FWHM of the spectrometer resolution function in $\mathrm{eV}$ at the $\mathrm{Si}(660)$ analyzer reflection order for different mask sizes, using a $\mathrm{Si}(111)$ pre-monochromator and a $\mathrm{Si}(311)$ channel-cut.

\begin{tabular}{lll}
\hline Mask size & $2 z=75 \mathrm{~mm}$ & $2 z=140 \mathrm{~mm}$ \\
\hline $40 \mathrm{~mm}$ & $0.50 \pm 0.01$ & $0.53 \pm 0.01$ \\
$60 \mathrm{~mm}$ & $0.65 \pm 0.02$ & $0.73 \pm 0.03$ \\
$80 \mathrm{~mm}$ & $0.86 \pm 0.03$ & $1.03 \pm 0.06$ \\
\hline
\end{tabular}

monochromator, and $\mathrm{Si}(111)$ pre-monochromator augmented by a $\mathrm{Si}(311)$ channel-cut monochromator] in Table 3.

3.1.4. Overall energy resolution. Tables 4 and 5 report the overall measured energy resolution based on the full width at half-maximum (FWHM) of quasi-elastic lines measured off a thin polymer foil for the most commonly used monochromator and spectrometer settings.

To further increase the overall resolving power of the spectrometer while conserving the large solid angle, one can take advantage of the fact that the dispersion across a spherically bent crystal analyzer can be compensated for using an off-focus geometry as was shown recently by Honkanen et al. (2014b). In comparison with Honkanen et al., however, the off-focus condition with the XRS spectrometer described here is achieved by displacing the analyzer crystals along the scattered beam direction (as opposed to displacing the area detector). Obviously, the off-focus geometry comes along with a loss of spatial resolution of the spectrometer and is thus not suitable for samples contained in highly complex sample 


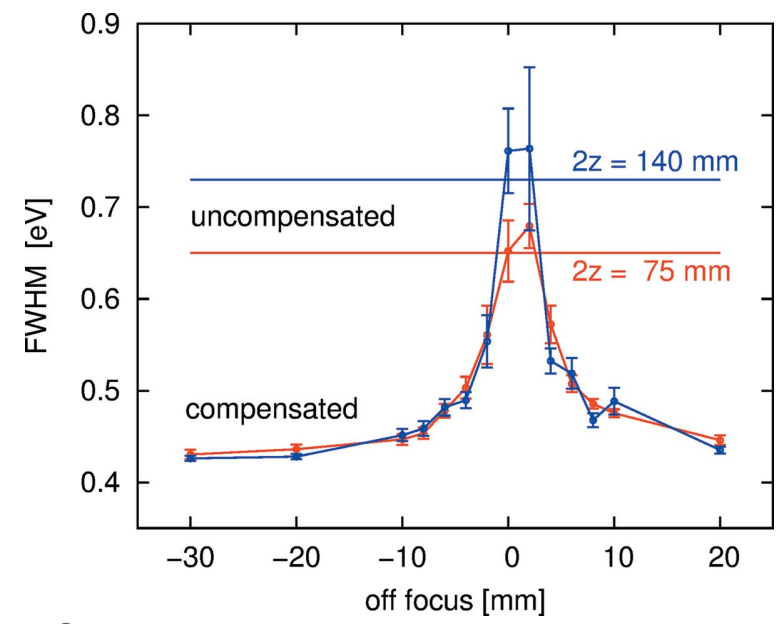

Figure 3

Measured FWHM of the spectrometer resolution function for different off-focus geometries after dispersion compensation (Honkanen et al., $2014 b$ ), in which analyzer crystals are displaced along the scattered beam direction to create a finite-sized focal spot on the detector and correcting for the dispersion pixel-by-pixel within that focal spot. The used analyzer openings were $80 \mathrm{~mm}$, defined with lead masks with circular apertures. Horizontal lines depict the corresponding resolution function without dispersion compensation.

environments. Fig. 3 shows the measured FWHM of resolution functions for different off-focusing geometries using the $\mathrm{Si}(660)$ reflection at $9.7 \mathrm{keV}$. The compensation was achieved by a pixel-by-pixel analysis of the intensities recorded for each individual analyzer crystal for an overall improvement of the resolving power of almost a factor of two.

\subsection{Detector housing and collimator}

Due to the small scattering cross section for non-resonant XRS, attention has to be paid to minimize the background due to stray scattering. The main sources of background noise are spurious scattering from the sample environment and/or air in the direct and scattered beam path. As mentioned earlier, each of the six modules of analyzer crystals is housed in a lightweight composite carbon fiber chamber operated in vacuum. In addition, the scattered radiation from the sample is collimated individually for each of the analyzer crystals using $10 \mathrm{~mm}$-long collimating aluminium apertures at the entrance of the analyzer modules (coined noses). The design of these combined collimating entrance windows and detector assembly is depicted in Fig. 4. The use of this individual collimation scheme increases the signal-to-noise ratio significantly.

\subsection{Sample goniometer and sample environments}

The sample goniometer is a modular tower with six degrees of freedom for sample orientation. The order of the motors from bottom to top is $R_{z}(\Theta), R_{x}(\Xi), R_{y}(\Phi), x, y, z$. The distance from the surface of the support to the center of rotation is $220 \mathrm{~mm}$ providing ample space for various sample environments.

A standard low-vacuum sample chamber ( $\sim 1 \mathrm{mbar})$ is available for small samples, such as single crystals and a) $140 \mathrm{~mm}$ nose

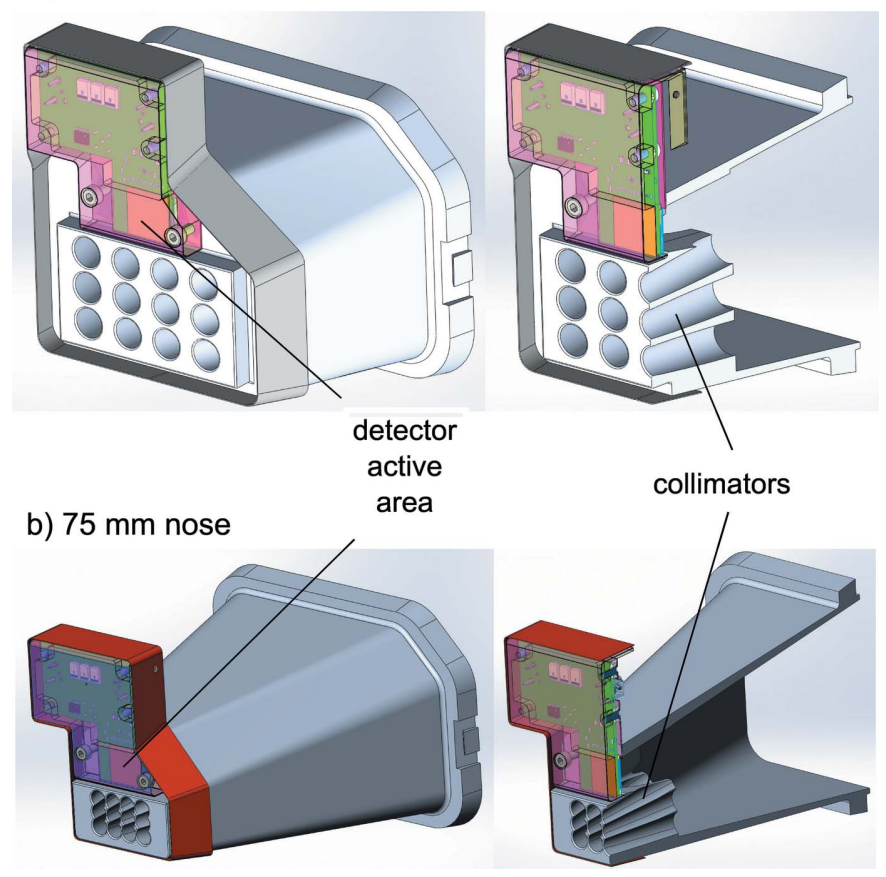

Figure 4

Sketch of the combined detector housing and collimators (coined noses) for the scattered X-ray photons as seen from the perspective of the analyzer crystals. Two different designs $(75 \mathrm{~mm}$ and $140 \mathrm{~mm}$ sample-todetector distance) exist for two different compromises between achievable energy resolution and available space for the sample environment.

powders, and samples contained in small containers, such as capillaries. A He exchange-gas cryostat to reach temperatures down to approximately $4 \mathrm{~K}$ is also available (van der Linden et al., 2016). Inside the cryostat, the samples are enclosed in a Be dome allowing for a large solid angle of collection. Highpressure conditions can be reached with two dedicated panoramic diamond anvil cells available via the ESRF's Sample Environment Support Service. Several other sample environments, such as a high-temperature furnace or hot air blower ( $T$ up to $1000 \mathrm{~K}$ ), or a cryostream blower ( $T$ down to $100 \mathrm{~K}$ ), are also accessible via the same service. Soft matter samples often suffer from radiation damage due to the high brilliance of the beamline. To study liquid samples under well defined thermodynamic conditions while simultaneously preventing damage due to exposure to radiation, we developed a compact liquid flow cell (Sahle et al., 2015a, 2016b). Fig. 5 shows how the He exchange-gas cryostat with a Be dome and a panoramic diamond anvil cell are typically mounted on the sample support. The dome allows for collection of data by all spectrometer modules, but the diamond anvil cell geometry restricts the useful modules to the vertical scattering plane.

\section{Data analysis and typical detector image}

The data collected by the Maxipix detectors are saved as binary files, and each file contains a composite image of all six detectors tiled in a $3 \times 2$ manner. X-rays reflected by each analyzer crystal are focused onto individual spots on the area 


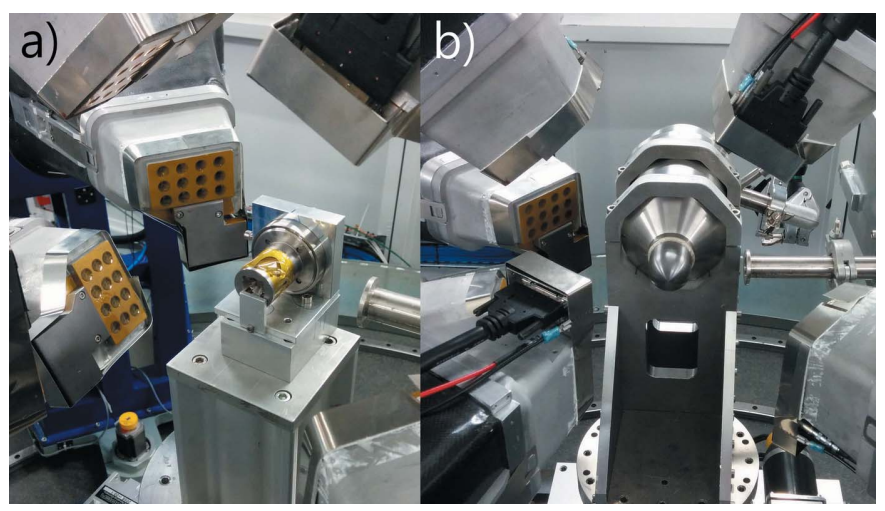

Figure 5

(a) Panoramic diamond anvil cell and (b) He exchange-gas cryostat mounted on the sample support, with noses of $2 z=140 \mathrm{~mm}$.

detector (12 per detector). For a point source, the size of the foci on the detector is of the order of a single detector pixel $(55 \mu \mathrm{m} \times 55 \mu \mathrm{m})$. Fig. 6 shows three examples of typical detector images from extended sources (see below for details). Examples for possible choices of regions of interest (ROIs) are shown as red dashed rectangles in the images. It is possible, before an image acquisition, to define these ROIs in the control software spec (spec, a software for instrument control and data acquisition; Certified Scientific Software, Cambridge, MA, USA, http://www.certif.com), after which the number of counted photons within that ROI appear in a corresponding counter and are saved in the datafile. However, in many cases the images contain much more information and often the analysis can be significantly improved by processing the saved images. For this purpose, a Python-based open source software (XRStools) has been developed and is described in detail elsewhere (Sahle et al., 2015b). The software allows for selection of ROIs in various ways: automatically based on a script for image analysis or the user can define the ROIs manually (rectangular, linear or polygon ROIs). XRStools then integrates over the ROIs and yields a)

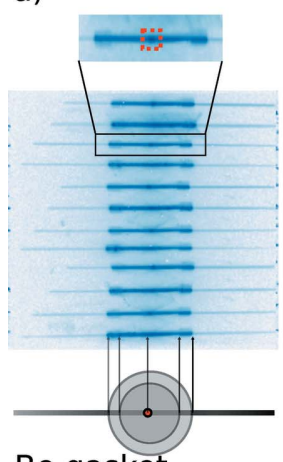

Be gasket b)

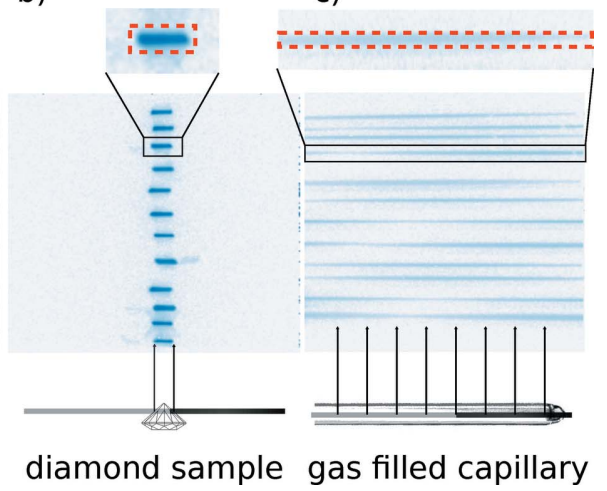

Figure 6

Typical detector images from the VU module for different samples. $(a)$ Scattering from a sample contained inside a diamond anvil cell, $(b)$ a diamond sample and $(c)$ scattering from a gas-phase sample contained inside a long quartz-glass capillary. The red dashed rectangles in the zoomed-in sections (top row) indicate possible choices for ROIs for the respective samples. final spectra that are normalized to the intensity monitor and averaged over repeated measurements. The results can be saved in an ASCII data file or can be processed further using XRStools.

\subsection{Focusing property: direct space imaging}

One of the powerful features of the presented XRS spectrometer is its ability to capture real-space information based on the so-called direct tomography technique [see Huotari et al. (2011) for details]. This greatly facilitates XRS measurements in complex sample environments, since scattering from the environment can be efficiently removed by selecting ROIs such that they contain only detector pixels that record scattering from the sample. This has led to a considerable improvement of the data quality of experiments utilizing, for example, diamond anvil pressure cells or chemical reactor cells, as well as containers of any kind. Moreover, the technique can reveal spatial information in naturally heterogeneous samples using XRS as contrast for the according image (Huotari et al., 2011).

Several typical detector images are depicted in Fig. 6, namely $(a)$ an image of a sample contained inside a diamond anvil cell, $(b)$ an image from a small diamond sample, and (c) a gas-phase sample ( $>15 \mathrm{~mm}$ beam path) contained inside a quartz-glass capillary. The small red dashed rectangles on the detector images illustrate possible ROIs for the respective samples. All images from Fig. 6 were taken with the VU module, recording scattering at approximately $90^{\circ}$ scattering angle in the vertical scattering plane. Small schematic drawings below the detector images are intended to describe the used geometries.

\section{Performance}

As the new spectrometer is an upgrade of the previous XRS spectrometer at beamline ID16 of the ESRF (Verbeni et al., 2009), obviously one should directly compare data from the old and new spectrometer. Such a comparison is shown for a spectrum of pure water in Fig. 7. At ID16, the spectrum was measured from a free-flowing liquid water column and scattering was recorded by nine spherically bent $\mathrm{Si}(660)$ analyzer crystals at a mean scattering angle of $36^{\circ}$ in the horizontal plane. For the data collection with the new spectrometer at ID20, we utilized the miniature liquid-flow cell mentioned earlier and $48 \mathrm{Si}(660)$ analyzer crystals in forward-scattering geometry. The arrangement of the analyzer chambers was with mean scattering angles at $35^{\circ}$ for the VD, VU, HR and HL chambers. The recorded intensities are presented in counts $\mathrm{s}^{-1}$ to grant direct comparison; however, the data from ID16 were collected for $120 \mathrm{~min}$ whereas the data taken with the new spectrometer were collected within $5 \mathrm{~min}$.

Figs. 8 and 9 show two further examples of data taken with the new spectrometer. In Fig. 8 we show spectra of liquid acetic acid, a simple carboxylic acid, measured over a broad energy-loss regime to cover the characteristic valence electron excitation regime as well as the carbon and oxygen $K$-edges. 


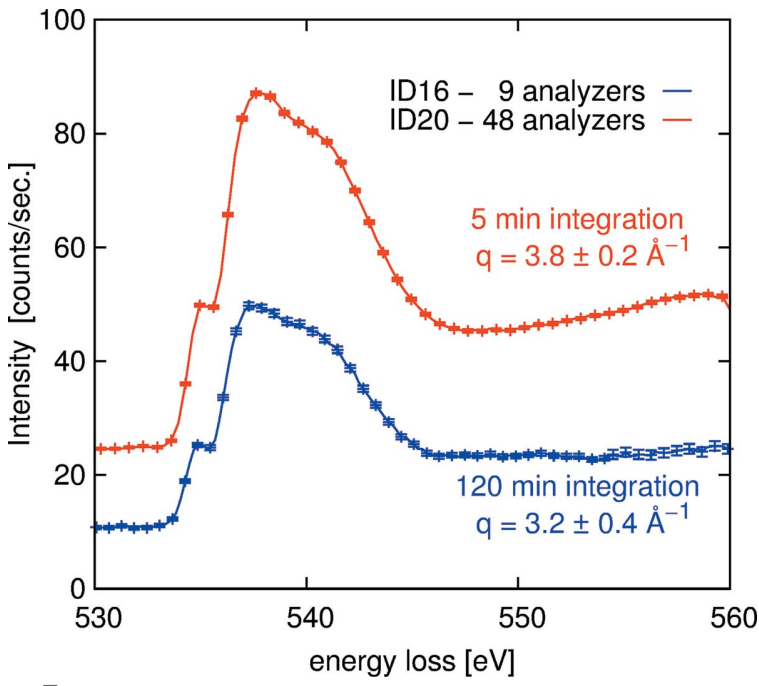

Figure 7

Comparison of raw data of liquid water taken with the nine-element spectrometer at ID16 and the new multi-analyzer spectrometer of ID20. For the data from the new spectrometer, we averaged over crystal analyzers with similar momentum transfer as used when collecting data with the old ID16 spectrometer. Intensity is the average per one analyzer.

The data are shown as raw data averaged over low $(q=3.8 \pm$ $\left.0.6 \AA^{-1}\right)$, intermediate $\left(q=6.8 \pm 0.5 \AA^{-1}\right)$ and high $(q=9.0 \pm$ $0.5 \AA^{-1}$ ) momentum transfers. For these averages we used individual signals from 36 (low $q$ ), 12 (intermediate $q$ ) and 24 (high $q$ ) $\operatorname{Si}(660)$ crystal analyzers, respectively. The overall measurement duration for the shown data was $100 \mathrm{~min}$, where most of the time was spent scanning the carbon and oxygen near-edge regions. The inset of the figure presents a closer view of the oxygen $K$-edge showing the excellent statistical accuracy of the data.

In Fig. $9(a)$ we show raw data from a highly oriented pyrolytic graphite (HOPG) sample. The sample was oriented such that the momentum transfer in the vertical scattering

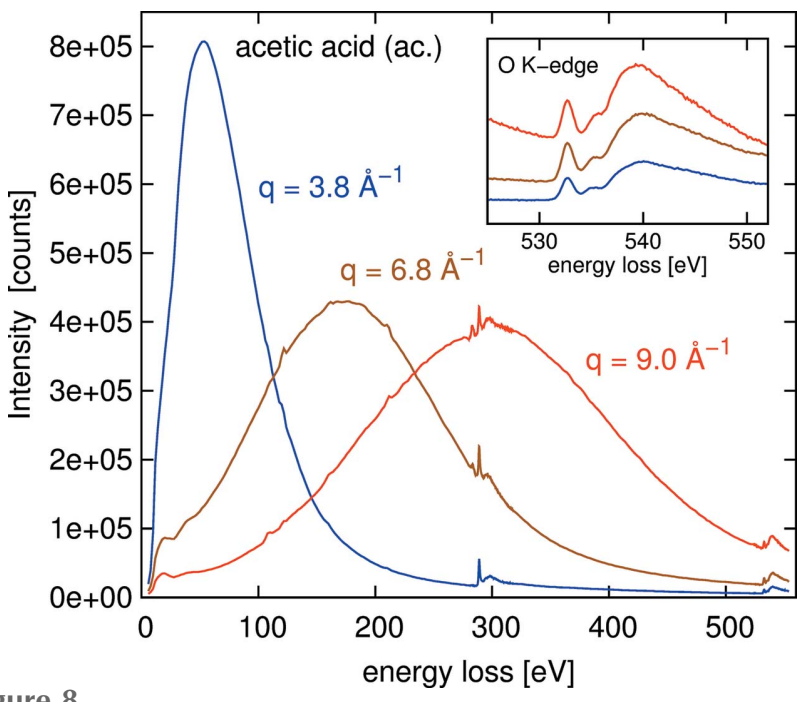

Figure 8

XRS spectra of liquid acetic acid over a large energy-loss range for three different momentum transfers. The inset shows a zoom into the energyloss region of the oxygen $K$-edge.
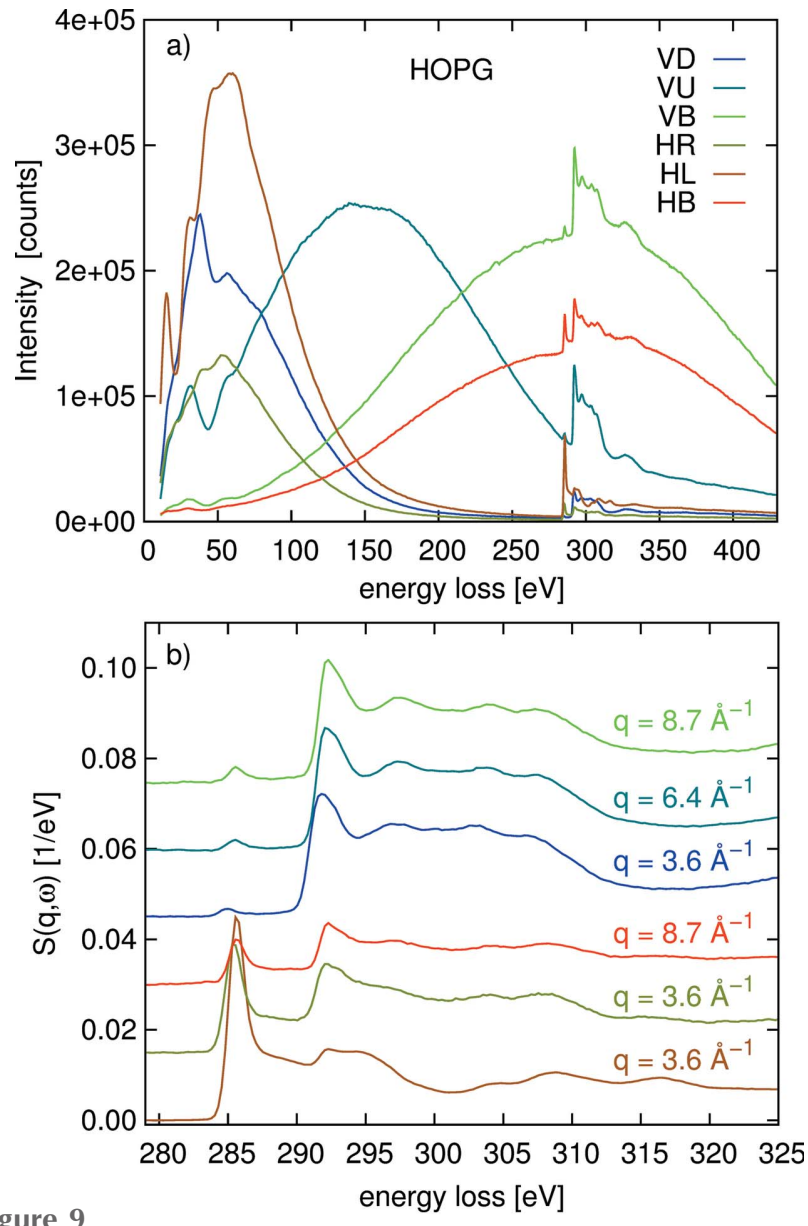

Figure 9

(a) Raw data from a HOPG sample over a large energy-loss range. The shown data were averaged module-by-module, i.e. the shown spectra are averages over signals from 12 analyzer crystals. (b) Carbon $K$ near-edge data after background subtraction and normalization of the data shown in part $(a)$. The spectra depend strongly on the scattering angle because of the anisotropy of HOPG.

plane is parallel to the sample's $a b$ plane. The data shown are averages over each of the analyzer modules, i.e. averages over scattering signals from 12 analyzer crystals. For the data shown in the figure, $8 \mathrm{~min}$ were spent for the collection of data of the large overview scan (spanning energy losses from 10 to $440 \mathrm{eV}$ energy loss) and $30 \mathrm{~min}$ for a fine scan around the carbon $K$ edge. Knowledge of the full loss spectrum greatly facilitates the background removal and normalization to absolute scale. In Fig. $9(b)$, the near-edge of the carbon $K$-edge is shown after post-processing using the XRStools package. The background was subtracted using parameterized Pearson-VII functions and normalization to absolute scale was achieved as described by Sahle et al. (2015b). The error bars for the respective plots are of the order of the line width.

\section{Conclusions and outlook}

We have presented a new experimental end-station for X-ray Raman scattering spectroscopy at port ID20 of the ESRF. The design of this new spectrometer was optimized to maximize the covered solid angle of detection while retaining a 
maximum amount of flexibility to account for complicated sample environments. We described the key characteristics of the spectrometer and depicted the use of pixelated area detectors that immensely facilitate the data analysis for experiments involving the mentioned complicated sample environments.

Future developments may include the optimization of the energy resolution while retaining the imaging capabilities of the analyzer crystals, extending the used range of primary energies to larger values, and the development of new sample environments to facilitate, for example, the in situ study of energy materials and catalytic reactions.

\section{Acknowledgements}

We kindly thank the ESRF for financing the UPBL6 project as part of the ESRF Upgrade Phase I. We kindly acknowledge M. A. Schroer, P. Degen and C. Sternemann for making available the ID16 data. This work has been supported by the Academy of Finland (grants 1259526 and 1283136).

\section{References}

Bergmann, U., Di Cicco, A., Wernet, P., Principi, E., Glatzel, P. \& Nilsson, A. (2007). J. Chem. Phys. 127, 174504.

Caciuffo, R., van der Laan, G., Simonelli, L., Vitova, T., Mazzoli, C., Denecke, M. A. \& Lander, G. H. (2010). Phys. Rev. B, 81, 195104.

Cai, Y. Q., Chow, P., Chen, C. C., Ishii, H., Tsang, K. L., Kao, C. C., Liang, K. S. \& Chen, C. T. (2003). Energy, 153, 50.

Conrad, H., Lehmkühler, F., Sternemann, C., Sakko, A., Paschek, D., Simonelli, L., Huotari, S., Feroughi, O., Tolan, M. \& Hämäläinen, K. (2009). Phys. Rev. Lett. 103, 218301.

Ding, Y., Chen, C. C., Zeng, Q., Kim, H. S., Han, M. J., Balasubramanian, M., Gordon, R., Li, F., Bai, L., Popov, D., Heald, S. M., Gog, T., Mao, H. K. \& van Veenendaal, M. (2014). Phys. Rev. Lett. 112, 056401.

Egerton, R. F. (2011). Electron Energy-Loss Spectroscopy in the Electron Microscope. Springer Science and Business Media.

Fister, T. T., Seidler, G. T., Wharton, L., Battle, A. R., Ellis, T. B., Cross, J. O., Macrander, A. T., Elam, W. T., Tyson, T. A. \& Qian, Q. (2006). Rev. Sci. Instrum. 77, 063901.

Galambosi, S., Knaapila, M., Soininen, J. A., Nygård, K., Huotari, S., Galbrecht, F., Scherf, U., Monkman, A. P. \& Hämäläinen, K. (2006). Macromolecules, 39, 9261-9266.

Gordon, R. A., Haverkort, M. W., Gupta, S. S. \& Sawatzky, G. A. (2009). J. Phys. Conf. Ser. 190, 012047.

Gordon, R. A., Seidler, G. T., Fister, T. T., Haverkort, M. W., Sawatzky, G. A., Tanaka, A. \& Sham, T. K. (2007). Europhys. Lett. 81, 26004.

Hämäläinen, K., Galambosi, S., Soininen, J. A., Shirley, E. L., Rueff, J.-P. \& Shukla, A. (2002). Phys. Rev. B, 65, 155111.

Hiraoka, N., Fukui, H. \& Okuchi, T. (2016). High. Press. Res. 36, 250261.

Hiraoka, N., Fukui, H., Tanida, H., Toyokawa, H., Cai, Y. Q. \& Tsuei, K. D. (2013). J. Synchrotron Rad. 20, 266-271.

Honkanen, A.-P., Verbeni, R., Simonelli, L., Moretti Sala, M., AlZein, A., Krisch, M., Monaco, G. \& Huotari, S. (2014b). J. Synchrotron Rad. 21, 762-767.

Honkanen, A.-P., Verbeni, R., Simonelli, L., Moretti Sala, M., Monaco, G. \& Huotari, S. (2014a). J. Synchrotron Rad. 21, 104-110.

Huotari, S., Pylkkänen, T., Soininen, J. A., Kas, J. J., Hämäläinen, K. \& Monaco, G. (2012). J. Synchrotron Rad. 19, 106-113.

Huotari, S., Pylkkänen, T., Verbeni, R., Monaco, G. \& Hämäläinen, K. (2011). Nat. Mater. 10, 489-493.
Huotari, S., Suljoti, E., Sahle, C. J., Rädel, S., Monaco, G. \& de Groot, F. M. F. (2015). New J. Phys. 17, 043041.

Inkinen, J., Niskanen, J., Talka, T., Sahle, Ch. J., Müller, H., Khriachtchev, L., Hashemi, J., Akbari, A., Hakala, M. \& Huotari, S. (2015). Sci. Rep. 5, 15851.

Inkinen, J., Sakko, A., Ruotsalainen, K. O., Pylkkänen, T., Niskanen, J., Galambosi, S., Hakala, M., Monaco, G., Huotari, S. \& Hämäläinen, K. (2013). Phys. Chem. Chem. Phys. 15, 9231-9238.

Juurinen, I., Pylkkänen, T., Ruotsalainen, K. O., Sahle, C. J., Monaco, G., Hämäläinen, K., Huotari, S. \& Hakala, M. (2013). J. Phys. Chem. B, 117, 16506-16511.

Juurinen, I., Pylkkänen, T., Sahle, C. J., Simonelli, L., Hämäläinen, K., Huotari, S. \& Hakala, M. (2014). J. Phys. Chem. B, 118, 87508755.

Krisch, M. H., Sette, F., Masciovecchio, C. \& Verbeni, R. (1997). Phys. Rev. Lett. 78, 2843-2846.

Lee, S. K., Lin, J. -F., Cai, Y. Q., Hiraoka, N., Eng, P. J., Okuchi, T., Mao, H. -k., Meng, Y., Hu, M. Y., Chow, P., Shu, J., Li, B., Fukui, H., Lee, B. H., Kim, H. N. \& Yoo, C. -S. (2008). Proc. Natl Acad. Sci. USA, 105, 7925-7929.

Linden, P. J. van der, Moretti Sala, M., Henriquet, C., Rossi, M., Ohgushi, K., Fauth, F., Simonelli, L., Marini, C., Fraga, E., Murray, C., Potter, J. \& Krisch, M. (2016). Rev. Sci. Instrum. 87, 115103.

Llopart, X., Ballabriga, R., Campbell, M., Tlustos, L. \& Wong, W. (2007). Nucl. Instrum. Methods Phys. Res. A, 581, 485-494.

Mao, W. L., Mao, H. K., Eng, P. J., Trainor, T. P., Newville, M., Kao, C. C., Heinz, D. L., Shu, J., Meng, Y. \& Hemley, R. J. (2003). Science, 302, 425-427.

Mattila, A., Soininen, J. A., Galambosi, S., Huotari, S., Vankó, G., Zhigadlo, N. D., Karpinski, J. \& Hämäläinen, K. (2005). Phys. Rev. Lett. 94, 247003.

Miedema, P. S., Ngene, P., van der Eerden, A. M. J., Weng, T., Nordlund, D., Sokaras, D., Alonso-Mori, R., Juhin, A., de Jongh, P. E. \& de Groot, F. M. F. (2012). Phys. Chem. Chem. Phys. 14, 5581-5587.

Mizuno, Y. \& Ohmura, Y. (1967). J. Phys. Soc. Jpn, 22, 445-449.

Moretti Sala, M. et al. (2017). In preparation.

Moretti Sala, M., Rossi, M., Boseggia, S., Akimitsu, J., Brookes, N. B., Isobe, M., Minola, M., Okabe, H., Rønnow, H. M., Simonelli, L., McMorrow, D. F. \& Monaco, G. (2014). Phys. Rev. B, 89, 121101.

Niskanen, J., Sahle, C. J., Juurinen, I., Koskelo, J., Lehtola, S., Verbeni, R., Müller, H., Hakala, M. \& Huotari, S. (2015). J. Phys. Chem. B, 119, 11732-11739.

Nyrow, A., Sternemann, C., Wilke, M., Gordon, R. A., Mende, K., Yavaş, H., Simonelli, L., Hiraoka, N., Sahle, Ch. J., Huotari, S., Andreozzi, G. B., Woodland, A. B., Tolan, M. \& Tse, J. S. (2014b). Contrib. Mineral. Petrol. 167, 1012.

Nyrow, A., Tse, J. S., Hiraoka, N., Desgreniers, S., Büning, T., Mende, K., Tolan, M., Wilke, M. \& Sternemann, C. (2014a). Appl. Phys. Lett. 104, 262408.

Pascal, T. A., Boesenberg, U., Kostecki, R., Richardson, T. J., Weng, T. C., Sokaras, D., Nordlund, D., McDermott, E., Moewes, A., Cabana, J. \& Prendergast, D. (2014). J. Chem. Phys. 140, 034107.

Ponchut, C., Rigal, J. M., Clément, J., Papillon, E., Homs, A. \& Petitdemange, S. (2011). J. Inst. 6, C01069.

Pylkkänen, T., Giordano, V. M., Chervin, J., Sakko, A., Hakala, M., Soininen, J. A., Hämäläinen, K., Monaco, G. \& Huotari, S. (2010). J. Phys. Chem. B, 114, 3804-3808.

Pylkkänen, T., Sakko, A., Hakala, M., Hämäläinen, K., Monaco, G. \& Huotari, S. (2011). J. Phys. Chem. B, 115, 14544-14550.

Rueff, J.-P. \& Shukla, A. (2010). Rev. Mod. Phys. 82, 847-896.

Sahle, C. J., Henriquet, C., Schroer, M. A., Juurinen, I., Niskanen, J. \& Krisch, M. (2015a). J. Synchrotron Rad. 22, 1555-1558.

Sahle, Ch. J., Kujawski, S., Remhof, A., Yan, Y., Stadie, N. P., Al-Zein, A., Tolan, M., Huotari, S., Krisch, M. \& Sternemann, C. (2016a). Phys. Chem. Chem. Phys. 18, 5397-5403.

Sahle, Ch. J., Mirone, A., Niskanen, J., Inkinen, J., Krisch, M. \& Huotari, S. (2015b). J. Synchrotron Rad. 22, 400-409. 
Sahle, Ch. J., Mirone, A., Vincent, T., Kallonen, A. \& Huotari, S. (2017b). J. Synchrotron Rad. 24, 476-481.

Sahle, C. J., Rosa, A. D., Rossi, M., Cerantola, V., Spiekermann, G., Petitgirard, S., Jacobs, J., Huotari, S., Moretti Sala, M. \& Mirone, A. (2017a). J. Synchrotron Rad. 24, 269-275.

Sahle, Ch. J., Sternemann, C., Schmidt, C., Lehtola, S., Jahn, S., Simonelli, L., Huotari, S., Hakala, M., Pylkkänen, T., Nyrow, A., Mende, K., Tolan, M., Hämäläinen, K. \& Wilke, M. (2013). Proc. Natl Acad. Sci. USA, 110, 6301-6306.

Sakko, A., Galambosi, S., Inkinen, J., Pylkkänen, T., Hakala, M., Huotari, S. \& Hämäläinen, K. (2011). Phys. Chem. Chem. Phys. 13, 11678-11685.

Schülke, W. (2007). Electron Dynamics by Inelastic X-ray Scattering. Oxford University Press.

Shieh, S. R., Jarrige, I., Wu, M., Hiraoka, N., Tse, J. S., Mi, Z., Kaci, L., Jiang, J.-Z. \& Cai, Y. Q. (2013). Proc. Natl Acad. Sci. USA, 110, 18402-18406.

Shvydko, Yu. (2004). X-ray Optics: High-Energy-Resolution Applications. Berlin Heidelberg: Springer-Verlag.

Soininen, J. A., Ankudinov, A. L. \& Rehr, J. J. (2005). Phys. Rev. B, 72, 045136.

Sokaras, D., Nordlund, D., Weng, T.-C., Mori, R. A., Velikov, P., Wenger, D., Garachtchenko, A., George, M., Borzenets, V.,
Johnson, B., Qian, Q., Rabedeau, T. \& Bergmann, U. (2012). Rev. Sci. Instrum. 83, 043112.

Sternemann, C., Sternemann, H., Huotari, S., Lehmkühler, F., Tolan, M. \& Tse, J. S. (2008). J. Anal. At. Spectrom. 23, 807-813.

Takagi, S. (1962). Acta Cryst. 15, 1311-1312.

Taupin, D. (1964). Bull. Soc. Fr. Mineral. Cristallogr. 87, 469.

Tse, J. S., Hanfland, M., Flacau, R., Desgreniers, S., Li, Z., Mende, K., Gilmore, K., Nyrow, A., Moretti Sala, M. \& Sternemann, C. (2014). J. Phys. Chem. C, 118, 1161-1166.

Tse, J. S., Yang, L., Zhang, S. J., Jin, C. Q., Sahle, Ch. J., Sternemann, C., Nyrow, A., Giordano, V., Jiang, J. Z., Yamanaka, S., Desgreniers, S. \& Tulk, C. A. (2011). Phys. Rev. B, 84, 184105.

Verbeni, R., Kocsis, M., Huotari, S., Krisch, M., Monaco, G., Sette, F. \& Vanko, G. (2005). J. Phys. Chem. Solids, 66, 2299-2305.

Verbeni, R., Pylkkänen, T., Huotari, S., Simonelli, L., Vankó, G., Martel, K., Henriquet, C. \& Monaco, G. (2009). J. Synchrotron Rad. 16, 469-476.

Wernet, Ph., Nordlund, D., Bergmann, U., Cavalleri, M., Odelius, M., Ogasawara, H., Näslund, L. A., Hirsch, T. K., Ojamäe, L., Glatzel, P., Pettersson, L. G. \& Nilsson, A. (2004). Science, 304, 995999.

Zhu, L. F., Wang, L. S., Xie, B. P., Yang, K., Hiraoka, N., Cai, Y. Q. \& Feng, D. L. (2011). J. Phys. B, 44, 025203. 